\title{
Diagnóstico e tratamento de cistos subcondrais em eqüinos: revisão de literatura
}

\author{
Diagnosis and treatment of subchondral cystic lesions in horses: \\ literature review
}

\section{Diagnóstico y tratamiento de los quistes subcondrales en caballos: revisión de la literatura}

\author{
Renata Bello Rossetti; ${ }^{1}$ Luís Cláudio Lopes Correia da Silva²
}

Departamento de Cirurgia da Faculdade de Medicina Veterinária e Zootecnia da Universidade de São Paulo (FMVZ/USP). São Paulo, SP, Brasil

\section{Resumo}

Objetivo: Analisar as diferentes formas de diagnóstico e tratamento de cistos subcondrais em eqüinos. Fontes Consultadas: Foram pesquisadas as bases de dados DEDALUS, PUBMED e o acervo da Biblioteca da Faculdade de Medicina Veterinária e Zootecnia da Universidade de São Paulo, São Paulo, SP, pelo período retrospectivo de 18 anos. Síntese dos Dados: As lesões císticas subcondrais (LCS), juntamente da osteocondrite dissecante, são consideradas manifestações comuns da osteocondrose nos eqüinos. As LCS no côndilo medial do fêmur são as mais freqüentemente encontradas, porém, podem ocorrer em múltiplos locais, incluindo: metacarpo, metatarso, rádio, escápula, úmero, tíbia, fêmur, carpo, tarso, falanges e sesamóides. Normalmente acometem animais jovens, em crescimento, com idade variando de seis meses a dois anos, que apresentam claudicação após iniciarem treinamento. Estudos clínicos indicam que o diagnóstico das LCS é possível por exame radiográfico, exame ultra-sonográfico, cintilografia e a ressonância magnética. A debridagem cirúrgica via artroscópica ou por artrotomia, o tratamento conservativo e o uso de corticóides intra-lesionais são citados como opções de tratamento. Conclusões: Embora existam modernas tecnologias - como a ressonância magnética e a cintilografia, capazes de realizar diagnósticos precoces dessas lesões -, o exame radiográfico ainda é a modalidade diagnóstica mais utilizada nesses casos, seguido pela ultra-sonografia. Com base no que foi revisto sobre os resultados obtidos com tratamento cirúrgico e conservativo, podemos considerar que são candidatos à curetagem cirúrgica os animais com sintomatologia clínica relacionada à LCS, de preferência por artroscopia, uma vez que dificilmente esses animais retornarão à atividade atlética sem o tratamento cirúrgico.

Palavras-chave: Cistos ósseos, etiologia. Cistos ósseos, diagnóstico. Cistos ósseos, terapia. Osteocondrite. Diagnóstico por imagem. Artroscopia. Equidae.

'Mestranda do Departamento de Cirurgia da FMVZ/USP. CRMV-SP 15.802

2Professor Doutor do Departamento de Cirurgia da FMVZ/USP. CRMV-SP 5.993 


\section{Introdução}

As lesões císticas subcondrais (LCS), juntamente da osteocondrite dissecante (OCD), são consideradas manifestações comuns da osteocondrose nos eqüinos. ${ }^{1}$ A osteocondrose é a alteração esquelética de desenvolvimento mais significativa nos eqüinos, causando grande impacto econômico na produção eqüina devido aos custos de tratamento e perda de animais por claudicações crônicas. ${ }^{2}$

São diversas as teorias já propostas sobre a etiologia e patogenia das lesões císticas subcondrais, as quais envolvem uma variedade de fatores, que incluem hereditariedade, desequilíbrios nutricionais, trauma biomecânico ${ }^{3,4}$ e presença de ostecondrose, ${ }^{4}$ ou até uma combinação entre esses elementos. ${ }^{5}$

Os cistos subcondrais podem ocorrer em múltiplos locais nos eqüinos, incluindo: metacarpo, metatarso, rádio, escápula, úmero, tíbia, fêmur, ossos do carpo e tarso, falange e sesamóides proximal e distal. $^{5}$

De acordo com relatos da literatura internacional, a maioria dos animais com LCS possuem idade entre um e três anos, e apresentam claudicação unilateral, porém, na rotina hospitalar do Serviço de Cirurgia de Grandes Animais da Faculdade de Medicina Veterinária e Zootecnia da Universidade de São Paulo (HOVET-FMVZ/USP), São Paulo, SP, observou-se em animais com idade mais avançada, após terem sido submetidos à atividade física.

A severidade da claudicação pode ser variável, dependendo da localização da lesão e das características inerentes ao defeito subcondral. ${ }^{5}$ Anestesias intra-articular ou regional podem ser úteis, para se localizar a origem da dor, e o exame radiográfico ainda é o mais utilizado rotineiramente para confirmar a presença do cisto.

As opções de tratamento para LSC dividemse em conservativo ou cirúrgico, ocorrendo controvérsia sobre a melhor terapêutica, tanto na literatura quanto na prática diária, seja entre autores internacionais, seja entre clínicos e cirurgiões brasileiros, não havendo uma unânime opção de tratamento.

\section{Etiologia e Patogenia}

As LCS são manifestações de osteocondrose, especialmente em animais jovens, com afecções bilaterais. ${ }^{6}$ Porém, é importante ressaltar que já foi descrita a ocorrência dessas lesões em animais mais velhos com lesão cartilagínea inicial, ${ }^{6}$ comprovando que essa doença possui etiologia multifatorial e que pode ser desencadeada por outra lesão primária, que não alteração de desenvolvimento.

Embora na rotina hospitalar do Serviço de Cirurgia de Grandes Animais (HOVET-FMVZ/USP), a manifestação da osteocondrose na forma de OCD seja muito mais freqüente do que as LCS, sabe-se que a predisposição da ocorrência de LCS na cartilagem da epífise de crescimento, em uma região de ossificação endocondral defeituosa, pode ser a mesma de uma OCD. Segundo a literatura, ao invés de a camada de cartilagem articular se destacar do osso subcondral, gerando a OCD, ela se aprisiona, sendo subseqüentemente reabsorvida e levando ao desenvolvimento do cisto subcondral. ${ }^{2,7}$ A cartilagem aprisionada necrosa e sua matriz permanecem desmineralizadas, não ocorrendo a migração de vasos sangüíneos e osteoclastos, impedindo a reparação do osso e cartilagem defeituosos. ${ }^{5}$

Foi demonstrada a presença de interleucina-6 (IL-6) em tecido fibroso de cisto subcondral e a produção de prostaglandina $\mathrm{E}_{2}\left(\mathrm{PGE}_{2}\right)$ por essas células quando cultivadas in vitro. Ao se promover a estimulação de cultura celular por agentes que atuam na reabsorção óssea, como o paratormônio, interleucina- $1 \beta$ (IL-1ß) e fator de necrose tumoral 2 alfa (TNF-2 $\alpha$ ), a síntese de IL- 6 já foi correlacionada com aumento simultâneo na produção de $\mathrm{PGE}_{2}$. (RECHENBERG et al. ${ }^{8}$ ). Desta forma, esses autores concluíram que a $\mathrm{PGE}_{2}$ em associação à IL-1ß e à IL-6 são as principais responsáveis pela reabsorção óssea em eqüinos acometidos com LCS, demonstrando que há provavelmente um componente inflamatório importante no desenvolvimento dessas lesões. 
Áreas susceptíveis a essas lesões estão sujeitas primariamente a forças de compressão, ${ }^{9}$ em que a articulação recebe máxima carga durante a fase de apoio, ${ }^{10}$ ao contrário dos locais acometidos por $\mathrm{OCD}$, que não sofrem carga. ${ }^{2}$

Em potros jovens, a presença de LCS de tamanho grande, ocupando porção significativa da articulação, comprova a teoria de que essas lesões se desenvolvem prematuramente em ossos em crescimento. Acredita-se que essas lesões aumentem de tamanho de forma gradativa enquanto o osso cresce, até que se comunicam com a articulação, remodelam e desaparecem; ou se mantêm de forma estática e assintomática. ${ }^{5}$ Lesões grandes e características de animais jovens não têm sido observadas na rotina do Serviço de Cirurgia de Grandes Animais (HOVET-FMVZ/USP), provavelmente devido ao fato de que raramente os animais jovens assintomáticos atendidos nesse Serviço são submetidos a exame radiográfico de controle. Presume-se que a comunicação do cisto com a articulação é criada por um evento traumático, que leva à manifestação clínica. ${ }^{5}$

Uma outra teoria da patogênese dos cistos subcondrais é proposta por Bramlage ${ }^{7}$ (1993), que sugeriu que um distúrbio inicial da ossificação endocondral (osteocondrose) é seguido de criação mecânica de lesão no osso subcondral devido ao bombeamento de líquido sinovial pelo defeito na superfície articular. A cartilagem defeituosa, que é espessada na área afetada como conseqüência da osteocondrose, eventualmente se racha, provavelmente devido a um trauma, promovendo pequena abertura que permite o bombeamento do líquido sinovial pelo defeito para o osso subcondral. O efeito mecânico do líquido sinovial no osso subcondral durante o apoio do membro causa reabsorção óssea e criação de lesão cística subcondral. A mecânica do fluido articular pode ditar o eventual tamanho da lesão, que aumenta gradualmente até que se obtenha um equilíbrio entre a pressão do líquido e a reabsorção do osso. Uma deposição óssea ao redor da circunferência da área lesionada, manifestada por esclerose subcondral, ocorre numa tentativa de se conter o insulto. ${ }^{7}$
Algumas evidências clínicas sugerem que o trauma pode ser uma das causas de LCS em eqüinos, incluindo-se a ocorrência de lesões em área de carga durante apoio, o diagnóstico em animais adultos e o ocasional alargamento lesional após debridagem cirúrgica. Um defeito na cartilagem articular e osso subcondral que permita infiltração de líquido sinovial não necessariamente precisa ter origem numa osteocondrose, mas sim em um trauma na articulação. ${ }^{5}$

\section{Sinais Clínicos}

Animais com LCS geralmente se apresentam com claudicação após iniciarem treinamento. Normalmente são animais jovens, em crescimento, com idade variando de seis meses a dois anos. ${ }^{2}$ É possível que isso ocorra principalmente nos animais que iniciam treinamento precocemente, como aqueles de corrida, por exemplo. Nos animais destinados a outros regimes de treinamento, como o salto clássico, concurso completo de equitação ou adestramento, normalmente o treinamento se inicia após o animal completar três anos de idade, momento em que pode ter ocorrido a resolução de distúrbios do desenvolvimento, sugerindo diferenças na evolução do processo e predisposição da ocorrência destas lesões. Entretanto, são necessários mais estudos com animais de outras atividades, que não corridas, para se averiguar a veracidade dessa hipótese.

$\mathrm{O}$ aparecimento e o grau de claudicação variam, dependendo da articulação afetada e uso do cavalo, ${ }^{2}$ mas geralmente são de grau 3 (escala de 0 a 5 ) ou menos. ${ }^{5}$ Em períodos que o animal é trabalhado, geralmente há piora da claudicação, ocorrendo melhora com repouso. Efusão articular, aumento de temperatura e dor local manifestam-se de forma variável. ${ }^{4}$ Esses sinais clínicos previamente descritos são bastante comuns nas afecções articulares em geral e, embora auxiliem na determinação do local lesionado, são incapazes, pela sua inespecificidade, de levar a um diagnóstico preciso sem a realização de exames complementares.

Watkins $^{2}$ (1999) e Baxter ${ }^{5}$ (1996) relataram que uma das causas de claudicação seria a dor presente no osso subcondral devido ao aumento da pressão 
intra-óssea no osso adjacente, que é decorrente do acúmulo de debris inflamatório e líquido sinovial. Outra causa seria a sinovite secundária à liberação de mediadores inflamatórios presentes na cavidade cística para o interior da articulação.

\section{Diagnóstico}

A origem da claudicação é facilmente reconhecida em cavalos que apresentam esse quadro de forma aguda e severa com efusão articular concomitante, ${ }^{5}$ entretanto, é pouco freqüente que os pacientes manifestem essa condição. ${ }^{2}$ No estudo realizado por Howard et al. ${ }^{11}$ (1995), no qual os animais exibiam lesões no côndilo femural, a maioria apresentava claudicação no membro acometido de grau 1 a 2, em uma escala de 0 a 5.

Outro fator a ser considerado é o teste de flexão da articulação acometida, que nesses casos pode ou não aumentar o grau de claudicação, ${ }^{5}$ sendo algumas vezes notada apenas relutância em se flexionar o membro. ${ }^{2}$

Desta forma, um exame de claudicação completo, com uso de bloqueios anestésicos perineurais e intra-articulares, é necessário para se descartar outras causas de claudicação e se localizar o sítio da dor. ${ }^{5}$ Entretanto, é importante ressaltar que a anestesia intra-articular pode confundir o diagnóstico se a lesão cística não se comunicar de forma suficiente com a articulação, dificultando a difusão do anestésico na cavidade cística ${ }^{7} \mathrm{e}$ causando, dessa forma, uma possível resposta falsonegativa ao bloqueio. Portanto, o clínico não deve descartar o emprego de meios diagnósticos auxiliares em casos de alterações articulares ao exame físico, independentemente da resposta ao bloqueio anestésico.

A seguir, serão descritos com mais detalhes os locais de ocorrência e as formas de diagnóstico, com ênfase nas técnicas mais indicadas para cada região.

\section{Articulação Fêmuro-tibial}

De acordo com a literatura consultada, as lesões císticas na porção proximal da tíbia são relatadas com baixa freqüência em relação aos cistos subcondrais no côndilo medial do fêmur. Entre todos os locais acometidos, esse é o mais freqüente nos eqüinos, principalmente no centro do côndilo e ocasionalmente adjacente à fossa intercondilar. ${ }^{2,5,6,12}$ Essa alta incidência não se repete nos animais atendidos no Serviço de Cirurgia de Grandes Animais (HOVETFMVZ/USP), onde há uma freqüência maior de diagnósticos de LCS na articulação do boleto.

A efusão sinovial é difícil de ser visualizada e detectada devido à anatomia da região, embora, segundo McIlwraith ${ }^{6}$ (2002), 50\% dos animais com LCS no côndilo femural apresentem efusão fềmuropatelar.

\section{Exame Radiográfico}

Segundo Watkins² (1999), as LCS são definitivamente diagnosticadas ao exame radiográfico, sendo melhor delineadas em exames de alta definição, nos quais se visualiza o padrão trabecular ósseo. Radiografias pouco expostas geralmente subestimam a extensão da lesão ou falham em identificá-las. Esta deve ser a principal razão pela qual ocorre uma baixa incidência de diagnóstico dessas lesões nos exames realizados no Serviço de Cirurgia de Grandes Animais (HOVET-FMVZ/USP), pois, infelizmente, em animais muito musculosos, a qualidade do exame radiográfico é inferior à necessária, porque não há disponibilidade de aparelhos radiográficos de alta definição.

No exame radiográfico, Howard ${ }^{11}$ et al. (1995) recomendam as projeções lateromedial, caudocranial e a lateromedial flexionada para articulação fềmurotibial. Além disso, o exame radiográfico deve ser realizado também no membro contralateral, pois, neste estudo, de 41 animais examinados com LCS, 27 apresentavam lesões bilaterais.

Quando visualizadas radiograficamente, as LCS no fềmur distal variam em forma e configuração. O cisto "clássico" se apresenta como uma área radiolucente circular ou ovalada, que se comunica com a superfície articular e possui esclerose óssea na porção periférica. Utilizando-se a projeção 
caudocranial é realizada a seguinte classificação: cistos tipo I têm menos de $10 \mathrm{~mm}$ de profundidade, forma de domo (meio-círculo), côncavo na superfície de apoio do côndilo e confluente com a superfície articular. Cistos tipo II provavelmente evoluem de cistos tipo I, e apresentam mais de $10 \mathrm{~mm}$ de profundidade, com formato em domo, cônico ou esférico. ${ }^{2}$

\section{Exame Ultra-sonográfico}

A superfície normal do osso subcondral é visualizada como uma linha regular hiperecogênica. Esta linha é interrompida quando há um cisto, sendo visualizada uma depressão no osso subcondral. Osteocondrose da tróclea femural pode ser diagnosticada com a visualização de espessamento da cartilagem e irregularidades no contorno do osso subcondral. Ossificação subcondral incompleta resulta em irregularidade da ecogenicidade do osso subcondral, como conseqüência da cartilagem parcialmente degenerada e ossificada na região da osteocondrose..$^{13-15}$ Cisto subcondral presente no côndilo medial do fêmur pode ser documentado com o paciente apoiando o membro acometido, utilizando-se acesso abaxial e o transdutor orientado proximalmente. $\mathrm{O}$ exame também pode ser realizado com o membro flexionado, utilizando-se acesso parasagital. ${ }^{15}$

A sensibilidade da ultra-sonografia é alta para se detectar defeitos subcondrais, sendo superior ao exame radiográfico. ${ }^{13}$ Embora aparentemente simples de ser realizado, e com disponibilidade de equipamentos adequados no País, o exame ultra-sonográfico articular exige, de forma essencial, um intenso treinamento e familiaridade com a técnica para que seja realizado de forma adequada e confiável. Desta forma, essa modalidade diagnóstica deve ser incentivada, suprindo a deficiência do exame radiográfico, principalmente nas articulações mais altas.

\section{Cintilografia}

Embora essa tecnologia não esteja disponível no Brasil, o uso de cintilografia nos casos de claudicação com diagnóstico indefinido é indicado, pois possibilita a identificação do local lesionado com alta precisão, apesar de algumas lesões crônicas nem sempre reterem contraste radioativo, podendo apresentar resultados inconsistentes. ${ }^{6}$

Assim, mesmo a cintilografia sendo importante ferramenta na detecção de inflamação, os exames radiográfico e ultra-sonográfico fornecem as informações sobre o tipo, tamanho e forma da lesão subcondral, não podendo ser substituídos, mesmo que haja disponibilidade futura dessa técnica.

\section{Carpo}

As lesões nessa região normalmente acometem o rádio distal e ossos do carpo e, embora diagnosticadas regularmente, apresentam pouca importância clínica. ${ }^{6}$ Casos de LCS em carpo não têm sido observados na rotina de atendimento do Serviço de Cirurgia de Grandes Animais (HOVETFMVZ/USP).

\section{Boleto}

Nas fontes de pesquisa consultadas, foi verificado que as LCS são pouco comuns nessa articulação e, quando ocorrem, acometem normalmente o metacarpo/metatarso distal, e com menos freqüência a porção proximal da primeira falange. ${ }^{3,6}$

Levantando-se os casos de LCS atendidos nos últimos dez anos no Serviço de Cirurgia de Grandes Animais (HOVET-FMVZ/USP), foi verificado maior incidência de acometimento dessa articulação, sendo também observada uma incidência alta nos sesamóides proximais (Figura 1), o que contraria a literatura consultada e necessita de investigação.

As lesões no boleto geralmente acometem o membro anterior e são raramente bilaterais. ${ }^{10}$ No estudo realizado por Hogan et al. ${ }^{3}$ (1997), foram avaliados 15 animais, observando-se predominância das lesões no côndilo medial do terceiro metacarpiano (13/15). Também foram notadas lesões no côndilo lateral e crista sagital. 


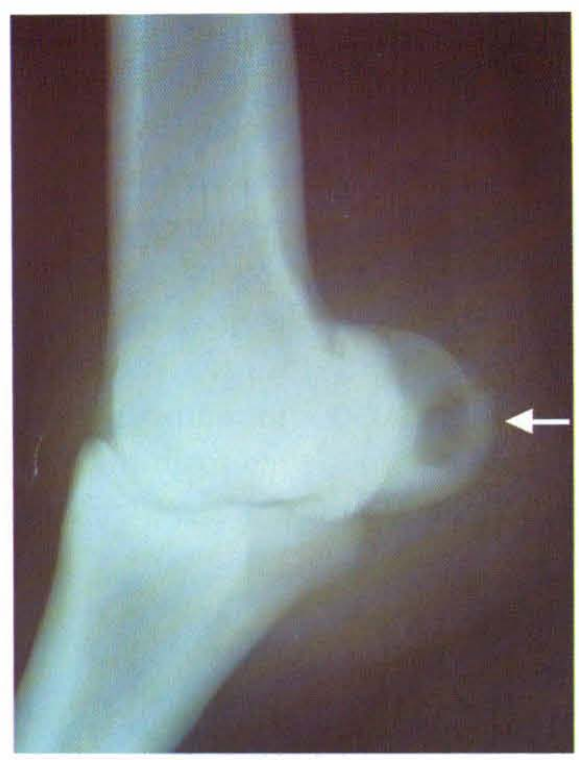

Figura 1 - Imagem radiográfica de lesão cística subcondral (seta) em sesamóide proximal de eqüino da raça Mangalarga de 13 anos de idade (Departamento de Cirurgia da FMVZ/USP)

\section{Exame Radiográfico}

O exame radiográfico confirma o diagnóstico, utilizando-se principalmente as projeções dorsopalmar e lateromedial em apoio. A projeção dorsopalmar angulada $\mathrm{em} 45^{\circ}$ pode fornecer mais detalhes em determinados casos.

Por ser uma região que permite maior facilidade ao exame físico e melhor qualidade ao exame radiográfico, é possível que haja uma tendência em diagnosticar cistos nessa região com maior freqüência quando comparada à articulação fêmuro-tíbio-patelar.

\section{Exame Ultra-sonográfico}

É possível verificar irregularidade do osso subcondral no côndilo do terceiro metacarpiano com a articulação flexionada, ${ }^{15}$ todavia, o exame radiográfico deve ser utilizado para confirmar os achados do exame ultra-sonográfico.

\section{Tomografia Computadorizada}

A tomografia computadorizada já é uma realidade em nossa escola para exames em pequenos animais e em potros jovens. Maiores investimentos ainda serão necessários para que essa tecnologia se torne viável à utilização em grandes animais, o que já ocorre nos Estados Unidos e na Europa. Nesses locais, o exame de tomografia computadorizada é utilizado em eqüinos para exame das regiões distais até as porções médias do rádio e da tíbia. Normalmente, essa técnica diagnóstica é utilizada em conjunto com exame radiográfico, e apresenta boa definição em casos de fraturas intra-articulares complexas, esclerose em osso subcondral induzida por estresse, fraturas não visualizadas no exame radiográfico e outras lesões subcondrais, como cistos ou defeitos sutis. ${ }^{14}$

\section{Ressonância Magnética}

Essa técnica diagnóstica tem sido introduzida de forma lenta na rotina de hospitais do hemisfério norte, sendo seu uso em eqüinos ainda restrito, principalmente, a centros de pesquisa, devido ao alto valor do equipamento e manutenção, o que acarreta um elevado custo por exame.

A ressonância magnética permite avaliação da estrutura óssea, tecidos moles e cartilagem articular, o que possibilita revelar lesões articulares de forma bastante precoce em relação ao exame radiográfico. ${ }^{16,17}$

Em estudo realizado por Zubrod et al. ${ }^{16}$ (2004), foram avaliados os prontuários de eqüinos atendidos com histórico de claudicação, sendo determinado por ressonância magnética o acúmulo de fluído anormal no osso subcondral, o qual não foi detectado por exame radiográfico.

\section{Articulação Interfalangeana Proximal}

As lesões nessa articulação geralmente ocorrem na porção distal da $1^{\mathrm{a}}$ falange, sendo descrita a ocorrência da doença em duas entidades distintas. A primeira, que ocorre em animais maduros, em que há presença de uma ou mais lesões associadas à osteoartrite; e a segunda ocorrendo em animais muito jovens, observando-se presença de múltiplas lesões císticas acompanhadas de 
osteoartrite severa, acometendo principalmente animais da raça Quarto-de-Milha. O diagnóstico é realizado por exame radiográfico, utilizando-se as projeções dorsopalmar e lateromedial. ${ }^{6}$

Casos de LCS em articulação interfalangeana proximal não têm sido observados na rotina de atendimento do Serviço de Cirurgia de Grandes Animais (HOVET-FMVZ/USP).

\section{Articulação Interfalangeana Distal}

As lesões ocorrem principalmente na porção proximal da $3^{\mathrm{a}}$ falange (Figura 2), e com menor freqüência na porção distal da $2^{\mathrm{a}}$ falange. ${ }^{6}$

Geralmente, essas lesões são consideradas clinicamente significativas quando apresentam comunicação com a cavidade articular, e esta pode ser verificada por exame radiográfico, xeroradiografias ou, ocasionalmente, por artrograma. ${ }^{6}$

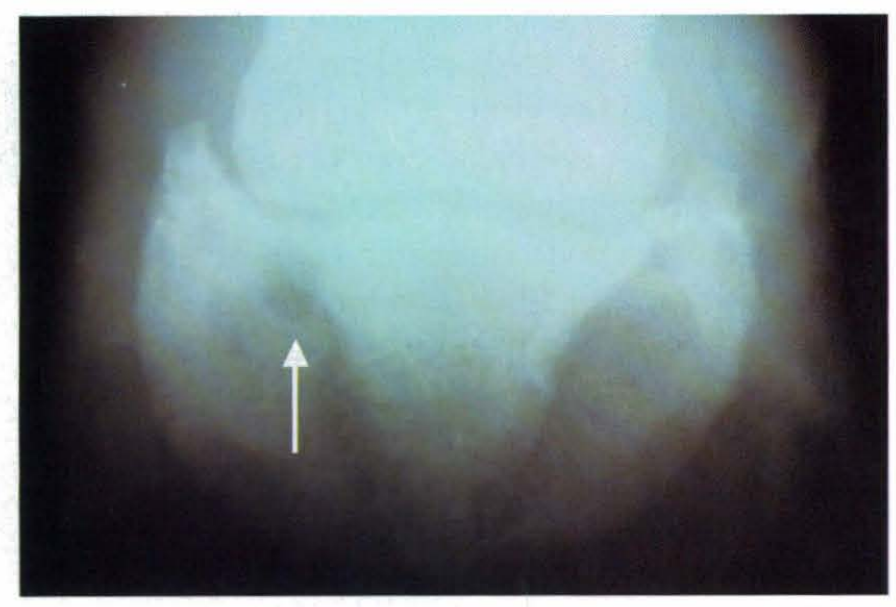

Figura 2 - Imagem radiográfica de lesão cística subcondral no aspecto proximal da $3^{\mathrm{a}}$ falange (seta) de eqüino da raça Mangalarga de sete anos de idade (Departamento de Cirurgia da FMVZ/USP)

\section{Articulação do Ombro}

As LCS nessa articulação ocorrem na cavidade glenóide, e estão comumente associadas à $\mathrm{OCD}$ na cabeça do úmero em animais jovens, sendo consideradas, portanto, uma manifestação da osteocondrose. Porém, as lesões císticas podem ocorrer na cavidade glenóide de maneira isolada. Normalmente, o animal acometido apresenta claudicação de membro anterior, geralmente associada à atrofia muscular e tamanho de casco diminuído em comparação com o membro nãoacometido. $\mathrm{O}$ exame radiográfico confirma a doença, e o bloqueio anestésico intra-articular confirma a importância clínica da lesão. ${ }^{6}$

\section{Articulação do Cotovelo}

As LCS normalmente envolvem o aspecto próximo-medial do rádio, mas ocasionalmente podem acometer o aspecto médio-distal do úmero. Os animais apresentam claudicação de membro anterior e o diagnóstico é realizado por exame radiográfico e, mais freqüentemente, por exclusão de outras fontes de claudicação. ${ }^{6}$

\section{Tratamento}

Segundo Story e Bramlage ${ }^{4}$ (2004), Baxter (1996) e Bramlage ${ }^{7}$ (1993), há muita controvérsia, não existindo consenso na literatura sobre a melhor forma de tratamento das LCS, sendo o mesmo observado na realidade brasileira. As recomendações variam de acordo com: articulação ou osso acometido; localização e tamanho da lesão; grau de acometimento da articulação; alterações articulares secundárias, como a doença articular degenerativa; duração da claudicação; idade do animal; uso do animal; e, principalmente, na nossa realidade, a situação financeira do proprietário.

$\operatorname{Baxter}^{5}$ (1996) relatou que há duas formas básicas de se abordar as LCS:

1- Tratamento conservativo: repouso com ou sem medicações intra-articulares e administração sistêmica de antiinflamatórios não-esteroidais; 2Debridamento cirúrgico.

A seguir, serão descritos com mais detalhes alguns relatos de literatura e os resultados obtidos em determinadas articulações. 


\section{Articulação Fêmuro-tibial}

$\operatorname{Baxter}^{5}$ (1996) citou que o uso de medicações intra-articulares - como o hialuronato de sódio e glicosaminoglicanos polissulfatados, ou a utilização sistêmica de antiinflamatórios não-esteroidais - tem sido empregado nos animais tratados de forma conservativa. Acredita-se que essas medicações atuem diminuindo o grau de sinovite secundária e aliviando a dor intra-óssea, porém, não se sabe sua capacidade de acelerar o remodelamento ósseo e promover resolução do defeito. Este mesmo autor também relatou sucesso limitado com uso de corticóide intra-articular em animais com LCS.

Segundo McIlwraith ${ }^{6}$ (2002), outros autores já relataram algum sucesso com adoção de tratamento conservativo nessa articulação, porém, os animais mais velhos apresentam pior prognóstico. Esse autor recomenda tratamento cirúrgico caso seja desejado cura visando ao desempenho atlético. Acredita-se que os benefícios da cirurgia provêm da remoção de material deletério presente na articulação, interrupção da liberação de mediadores inflamatórios e alteração na pressão intra-óssea. ${ }^{6}$ Ele ainda relatou que, embora em alguns casos possa ocorrer cura espontânea, o prognóstico para atividade atlética aumenta após a cirurgia de $20 \%$ a $75 \%$.

O tratamento cirúrgico objetiva a remoção do conteúdo do cisto, do osso subcondral esclerótico ao redor da lesão e da cartilagem, que não possui suporte ósseo adequado. Dependendo da articulação acometida, o tratamento pode envolver uso de artrotomia, artroscopia ou até acesso extra-articular. ${ }^{9}$

McIlwraith $^{6}$ (2002) relatou o uso de implante de osso esponjoso por meio de acesso extra-articular entre os anos de 1975 a 1978. Nos seis casos tratados dessa forma, nenhum apresentou cura para performance atlética, e o insucesso desta técnica foi atribuído à curetagem inadequada da borda do cisto e não-penetração no osso esclerosado. A partir de 1979, este mesmo autor utilizou o acesso intraarticular via artrotomia fêmuro-tibial. Com essa técnica, foram operados 42 animais que não apresentavam osteoartrite concomitante, e todos manifestaram melhora clínica, sendo 35 sadios ao retornarem à atividade atlética. Baxter ${ }^{5}$ (1996), no entanto, relatou não haver benefício do implante de osso esponjoso, ocorrendo reabsorção do implante 6 a 12 meses após o procedimento cirúrgico.

Em estudo realizado com eqüinos por Jackson et al. ${ }^{18}$ (2000), no qual se comparou a cicatrização de LCS com e sem uso de implante de osso esponjoso, confirmou-se não haver benefício dessa técnica, pois não foi observado, durante os seis meses do estudo, diferença na cicatrização da lesão. Tais autores afirmaram que a curetagem cirúrgica do cisto por via artroscópica ainda é o tratamento de eleição nesses casos.

Em estudo realizado com 41 animais apresentando LCS no côndilo medial do fêmur, tratados por via artroscópica por Howard et al. ${ }^{11}$ (1995), foi relatada a ocorrência de expansão da LCS após a cirurgia em $65 \%$ dos casos. Estes autores sugeriram que essa expansão ocorreu devido à perda da integridade da cartilagem articular e à pressão exercida pelo líquido sinovial no osso subcondral. Nesse estudo, também se verificou correlação positiva entre a perfuração com broca e a expansão observada no pós-operatório. Os autores acreditam que essa técnica pode aumentar a exposição do osso epifisário a mediadores inflamatórios e enzimas degradadoras - aumentando, dessa forma, a reabsorção óssea - tornando-a, portanto, contraindicada.

McIlwraith $^{6}$ (2002) também relatou que a realização de perfurações no osso subcondral é desaconselhada, pois acarreta em aumento da lesão no período pós-operatório, fato este também observado por Baxter ${ }^{5}$ (1996). O primeiro autor tem utilizado, após a curetagem do cisto, aplicação intralesional de $40 \mathrm{mg}$ de acetato de metilprednisolona (Depo-Medrol ${ }^{\circledR}$ ), o que tem evitado aumento da lesão posterior ao tratamento cirúrgico.

O uso intra-lesional de corticóide como tratamento de cisto ósseo unicompartimental em humanos jovens substitui a necessidade de curetagem 
cirúrgica. Acredita-se que este fármaco apresente efeitos nos componentes celulares do cisto e também atue como estímulo osteogênico, pois, após a aplicação, observa-se diminuição considerável da dor do paciente e o cisto é preenchido por novo tecido ósseo. ${ }^{5}$ McIlwraith $^{6}$ (2002) relatou que os achados de Rechenberg et al. ${ }^{8}$ (2001), da presença de mediadores da inflamação na parede do cisto subcondral, justificam a utilização de corticosteróides intra-lesionais.

A injeção intra-lesional de corticóide tem surgido, também em Medicina Veterinária, como uma forma de tratamento em substituição à curetagem cirúrgica e, apesar de não haver definição quanto às condições de sua aplicação, os resultados têm sido promissores.

Bodo et al. ${ }^{19}$ (2004) relataram a realização de mosaicoplastia com implante osteocondral por artrotomia ou artroscopia, para o tratamento de eqüinos com LCS, na articulação fêmuro-tibial e do boleto, os quais haviam sido tratados anteriormente de maneira conservativa com repouso e administração de corticóide intra-articular sem sucesso. Os implantes utilizados nesses animais eram coletados em formato cilíndrico, com 4,5 a 6,5 mm de diâmetro, do aspecto medial da tróclea do fêmur da articulação ipsi ou contra-lateral. Neste estudo, 9 dos 11 animais operados retornaram ao nível de atividade anterior à manifestação da lesão. Acredita-se que essa técnica seja um avanço em relação à cicatrização do cisto, porém, é necessário se levar em consideração o trauma cirúrgico, a dificuldade na recuperação, os riscos de infecção e o tempo necessário para que o animal volte à atividade após a realização de artrotomia, sendo, na opinião dos autores da presente revisão, uma alternativa para os casos refratários a outras opções de tratamento.

\section{Carpo}

O tratamento dos casos de LCS nessa articulação não tem sido sugerido na literatura, uma vez que a manifestação clínica não é comum. McIlwraith $^{6}$ (2002) recomendou o tratamento cirúrgico para lesões císticas no carpo que não apresentaram melhora em seis meses de tratamento conservativo.

\section{Boleto}

Segundo Bramlage ${ }^{7}$ (1993), as LCS presentes no metacarpo distal apresentam prognóstico reservado, independentemente do tratamento utilizado. Mcllwraith $^{6}$ (2002) recomendou o tratamento cirúrgico com curetagem do cisto, embora a técnica de artroscopia seja bem difícil, pois é necessário máxima flexão da articulação para se expor à comunicação do cisto com a superfície articular.

Em estudo realizado por Hogan et al. ${ }^{3}$ (1997) com 15 animais, $80 \%$ apresentaram cura, com retorno à atividade, após curetagem cirúrgica por via artroscópica.

Embora o uso intra-articular de corticóide não seja recomendado pela literatura, na rotina do Serviço de Cirurgia de Grandes Animais (HOVET-FMVZ/ USP) ele tem sido empregado nos casos em que não é aceito o tratamento cirúrgico pelo proprietário. Constituiu tratamento de caráter paliativo, com resultado temporário, considerando-se que a curetagem cirúrgica é a primeira opção (Figura 3).

\section{Articulação Interfalangeana Proximal}

As LCS presentes na porção distal da falange proximal podem responder bem a tratamento conservativo e permanecerem funcionalmente sadias. ${ }^{6}$

Nos animais jovens, que apresentam múltiplas lesões císticas associadas à osteoartrite severa, o prognóstico é ruim e o único tratamento indicado é a artrodese cirúrgica. ${ }^{5,6}$

\section{Articulação Interfalangeana Distal}

Há um consenso entre diversos autores de que a resposta dessas lesões ao tratamento conservativo é pobre. ${ }^{6}$ Story e Bramlage ${ }^{4}$ (2004) relataram sucesso de $91 \%$ nos animais com LCS na $3^{\mathrm{a}}$ falange tratados por curetagem da lesão por via artroscópica. É importante ressaltar que a curetagem dessa 

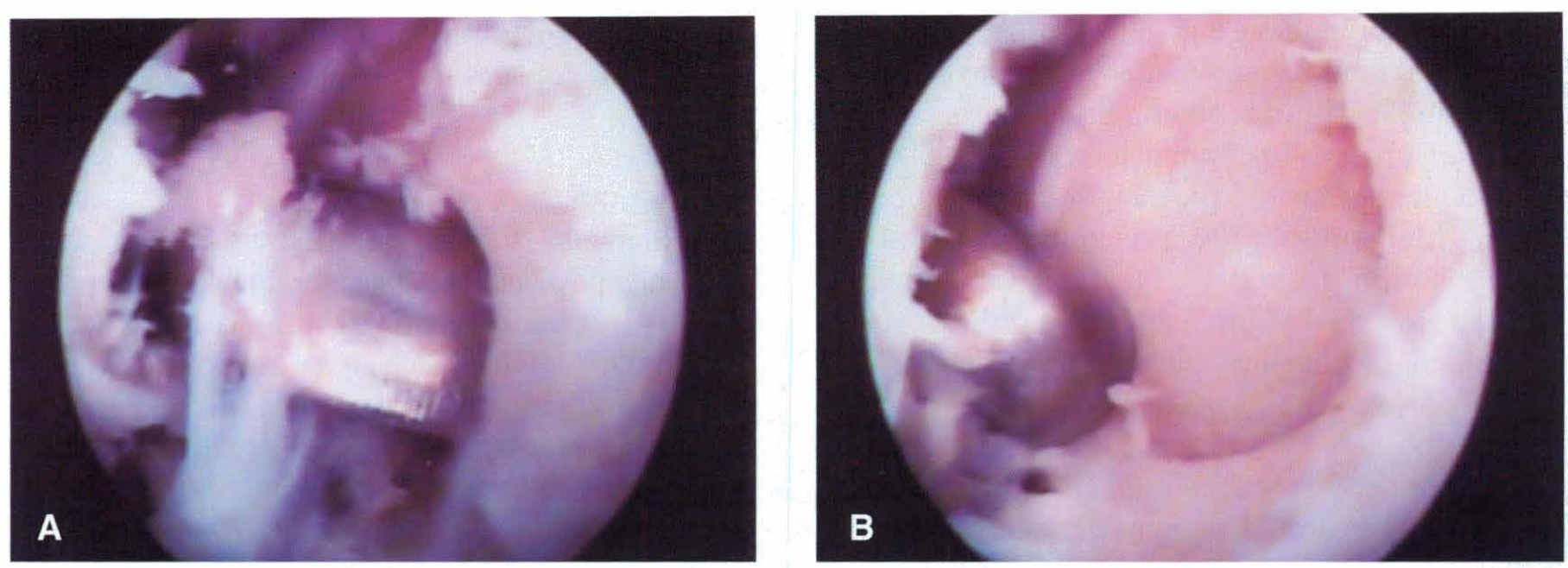

Figura 3 - Imagem artroscópica de curetagem de lesão cística subcondral em côndilo medial do $3^{\circledR}$ metacarpiano de eqüino da raça Mangalarga de sete anos de idade. A - Início da curetagem; B - Final da curetagem (Departamento de Cirurgia da FMVZ/USP)

articulação por artroscopia é bastante difícil devido ao restrito espaço articular disponível e limita-se a lesões localizadas no bordo dorso-proximal da terceira falange.

\section{Articulação do Ombro}

Segundo McIlwraith ${ }^{6}$ (2002), as LCS presentes no ombro não respondem a tratamento conservativo e a única opção é a cirurgia artroscópica com debridamento da lesão.

\section{Articulação do Cotovelo}

Bertone et al. ${ }^{20}(1986)$ relataram que eqüinos com LCS no cotovelo e tratados com curetagem cirúrgica por artrotomia apresentaram retorno à atividade atlética inicial e menor evidência de doença articular degenerativa, quando comparados a animais tratados de maneira conservativa.

\section{Conclusões}

Os estudos clínicos avaliados na presente revisão indicam que a forma mais comum de diagnóstico das lesões císticas subcondrais ainda é por exame radiográfico. Todavia, o exame ultra- sonográfico, de boa disponibilidade em situações de campo, e a ressonância magnética, não disponível na rotina clínica veterinária no Brasil, também podem ser utilizados. Há necessidade de mais investimentos em modernização dos aparelhos radiográficos no País. Também é fundamental proporcionar maior capacitação aos médicos veterinários, que atualmente utilizam o exame ultra-sonográfico para avaliação apenas das estruturas tendíneas e ligamentares do aparelho locomotor. Isso implicaria em incremento da capacidade diagnóstica em técnicas acessíveis aos proprietários.

Por meio de uma melhor capacidade diagnóstica, é possível que sejam realizados estudos nacionais mais precisos sobre a ocorrência de LCS em eqüinos, a qual parece diferir da descrita na literatura internacional.

Os animais com sintomatologia clínica relacionada à LCS são candidatos à curetagem cirúrgica, de preferência por artroscopia. Apesar de esse tratamento ser mais dispendioso e necessitar de mais tempo de repouso que o tratamento conservativo, acredita-se ser o mais indicado quando o objetivo é o retorno à atividade atlética. Contudo, os estudos utilizando administração intra-lesional de corticóides podem trazer mudanças nesse conceito. 


\section{Abstract}

Objective: To analyze the different diagnostic techniques and treatment options for subchondral cystic lesions in horses. Data Sources: DEDALUS and PUBMED databases and the collection of library of the Faculdade de Medicina Veterinária e Zootecnia da Universidade de São Paulo (School of Veterinary Medicine and Animal Science of the University of São Paulo), São Paulo, SP, Brazil, were searched, covering a retrospective period of 18 years. Data Synthesis: Subchondral cystic lesions (SCL) and osteochondritis dissecans (OCD) are common manifestations of osteochondrosis in horses. SCLs are most frequently seen in the medial femoral condyle, but they can occur at different sites, such as the metacarpus, metatarsus, radius, scapula, humerus, tibia, femur, carpus, tarsus, phalanges and sesamoids. Horses affected with SCLs are typically young, growing animals, mostly between 6 months and 2 years of age, presenting with lameness after the onset of training. According to clinical studies, SCLs can be diagnosed by radiographic examination, ultrasonographic examination, scintigraphy, and magnetic resonance imaging. Surgical debridement through arthroscopy or arthrotomy, conservative treatment and intralesional corticosteroids are mentioned as treatment options. Conclusions: Despite the modern technologies available, such as magnetic resonance imaging and scintigraphy, that enable early diagnosis of these lesions, radiology still remains the most popular diagnostic technique employed, followed by ultrasonography. Based on documented results of both, surgical and conservative treatments, horses presenting with clinical signs related to a SCL are potential candidates for surgical debridement, preferably through arthroscopic surgery, once such patients will probably not regain athletic soundness unless they are operated on.

Keywords: Bone cysts, etiology. Bone cysts, diagnosis. Bone cysts, therapy. Diagnostic imaging. Arthroscopy. Osteochondritis. Equidae.

\section{Resumen}

Objetivo: Analizar las diferentes formas de diagnóstico y tratamiento de los quistes subcondrales en caballos. Fuentes Consultadas: Fueron estudiadas las bases de datos DEDALUS, PUBMED, así como el archivo bibliográfico de la Biblioteca de la Facultad de Medicina Veterinaria y Zootecnia de la Universidade de São Paulo, São Paulo, SP, Brasil, durante el periodo retrospectivo de 18 años. Síntesis de los Datos: Las lesiones quísticas subcondrales (LQS) junto con la osteocondrosis disecante, son consideradas manifestaciones comunes de la osteocondrosis de los equinos. Las LQS en el cóndilo medial del fémur, son las lesiones que se encuentran con mayor frecuencia. No obstante, pueden ser encontradas en otros lugares como: metacarpo, metatarso, radio, escápula, húmero, tibia, fémur, carpo, tarso, falanges y sesamoides. Normalmente afectan a animales jóvenes, en crecimiento, con edad que varía entre los 6 meses y 2 años y que presentan claudicación después de iniciar la actividad deportiva. Estudios clínicos indican que el diagnóstico de las LQS se puede realizar a través de exámenes radiográficos, ecográficos, centellografia y resonancia magnética. El tratamiento quirúrgico vía artroscópica o por atrotomía; el tratamiento conservador y la utilización de corticoides intralesionales, son citados por diferentes autores como opciones posibles de tratamiento. Conclusiones: A pesar de que existen tecnologias modernas, como la resonancia y la centellografia, capaces de realizar diagnósticos precoces de las lesiones, el examen radiográfico continúa siendo la técnica mas utilizada para estos pacientes, seguido por la ecografía. En relación a los resultados obtenidos con el tratamiento quirúrgico y conservador, los animales con síntomas clinicos de LQS son considerados candidatos a curetaje quirúrgico, preferentemente por artroscopia, ya que dificilmente estos animales retornarán a su actividad atlética sin tratamiento quirúrgico.

Palabras-clave: Quistes óseos, etiología. Quistes óseos, diagnóstico. Quistes óseos, terapia. Diagnóstico por imagen. Artroscopia. Osteocondritis. Equidae. 


\section{Referências}

1. RIEGEL, R. J.; HAKOLA, S. E. The fetlock and pastern. In: _. Ilustrated atlas of clinical equine anatomy and common disorders of the horse. 4. ed. Marysville: Equistar, 2001. p. 82-102.

2. WATKINS, J. P. Osteochondrosis. In: AUER, J. A., STICK, J. A. Equine surgery. 2. ed. Philadelphia: Saunders, 1999. p. 765-778.

3. HOGAN, P. M. et al. Surgical treatment of subchondral cystic lesions of the third metacarpal bone: results in 15 horses (1986-1994). Equine Veterinary Journal, v. 29, p. 477-482, 1997.

4. STORY, M. R.; BRAMLAGE, L. R. Arthroscopic debridement of subchondral bone cyst in distal phalanx of 11 horses (1994-2000). Equine Veterinary Journal, v. 36, p. 356-360, 2004.

5. BAXTER, G. M. Subchondral cystic lesions in horses. In: McILWRAITH, W. C.; TROTTER, G. W. Joint disease in the horse. Philadelphia: Saunders, 1996. p. 384-396.

6. McILWRAITH, C. W. Diseases of joints, tendons, ligaments, and related structures. In: STASHAK, T. S. Adams' lameness in horses. 5. ed. Baltimore: Lippincott Williams \& Wilkins, 2002. p. 459-644.

7. BRAMLAGE, L. R. Osteochondrosis related bone cysts. In: ANNUAL CONVENTION OF THE AMERICAN ASSOCIATION OF EQUINE PRACTITIONERS, 39., 1993, San Antonio. Proceedings... Lexington: The American Association of Equine Practitioners, 1993. p. 83-85.

8. RECHENBERG, B. et al. Upregulation of mRNA of interleukin-1 and -6 in subchondral cystic lesions of four horses. Equine Veterinary Journal, v. 33, p. 143-149, 2001.

9. EASTER, J. L.; WATKINS, J. P. Osteochondrosis and physitis. In: COLAHAN, P. T. et al. Equine medicine and surgery. 5. ed. Saint. Louis: Mosby, 1999. p. $1475-1482$.
10. NIXON, A. J. Osteochondrosis and osteochondritis dissecans of equine fetlock. Compendium on Continuing Education for the Practicing Veterinarian, v. 12, p. 1463-1475, 1990.

11. HOWARD, R. D.; McILWRAITH, W. C.; TROTTER, G. W. Arthroscopic surgery for subchondral cystic lesions of the medial femural condyle in horses: 41 cases (1988-1991). Journal of the American Veterinary Medical Association, v. 206, p. 842-850, 1995.

12. TEXTOR, J. A. et al. Subchondral cystic lesions of the proximal extremity of the tibia in horses: 12 cases (1993-2000). Journal of the American Veterinary Medical Association, v. 218, p. 408-413, 2001.

13. DENOIX, J. M.; AUDIGIÉ, F. Imaging of the musculosketletal system in horses. In: HINCHCLIFF, K. W.; KANEPS, A. J.; GEOR, R. J. Equine sports medicine and surgery. Philadelphia: Saunders, 2004. p. 161-187.

14. PARK, R. D.; WRIGLEY, R. H.; STEYN, P.F. Equine diagnostic imaging. In: STASHAK, T. S. Adams' lameness in horses. 5. ed. Baltimore: Lippincott Williams \& Wilkins, 2002. p. 185-375.

15. DENOIX, J. M. Ultrasonographic examination in the diagnosis of joint disease. In: McILWRAITH, W. C.; TROTTER, G. W. Joint disease in the horse. Philadelphia: Saunders, 1996. p. 165-202.

16. ZUBROD, C. J. et al. Use of magnetic resonance imaging for indetifying subchondral bone damage in horses: 11 cases (1999-2003). Journal of the American Veterinary Medical Association, v. 224, p. 411-418, 2004.

17. WERPY, N. M. et al. Magnetic resonance imaging of the metacarpophalangeal joint in nine racehorses. In: AAEP FOCUS ON JOINTS MEETING, 2004, Louisville. Proceedings... Lexington: The American Association of Equine Practitioners, 2004. p. 56-66. 
18. JACKSON, W. A. et al. The effect of compacted cancellous bone grafting on the healing of subchondral bone defects of the medial femoral condyle in horses. Veterinary Surgery, v. 29, p. 8$16,2000$.

19. BODO, G.; HURTIG, M. B. Management of subchondral cystic lesions with osteochondral grafts.
In: AAEP FOCUS ON JOINTS MEETING, 2004, Louisville. Proceedings... Lexington: The American Association of Equine Practitioners, 2004. p. 200-201.

20. BERTONE, A. L. et al. Subchondral osseous cystic lesions of the elbow of horses: conservative versus surgical treatment. Journal of the American Veterinary Medical Association, v. 189 , p. $540-546,1986$. 